\title{
ON THE NATURE AND DYNAMICS OF THE SEISMOGENETIC SYSTEM OF SOUTH CALIFORNIA, USA: AN ANALYSIS BASED ON NON-EXTENSIVE STATISTICAL PHYSICS
}

\author{
Efstathiou A. ${ }^{1}$, Tzanis A. ${ }^{1}$ and Vallianatos F. ${ }^{2}$ \\ ${ }^{I}$ Department of Geophysics and Geothermy, Faculty of Geology and Geoenvironment, National \\ and Kapodistrian University of Athens, aefstathiou@geol.uoa.gr,atzanis@geol.uoa.gr \\ ${ }^{2}$ Laboratory of Geophysics and Seismology, Technological Educational Institute of Crete, Chania, \\ GR 73133,Crete,fvallian@chania.teicrete.gr
}

\begin{abstract}
We examine the nature of the seismogenetic system in South California, USA, by searching for evidence of non-extensivity in the earthquake record. We attempt to determine whether earthquakes are generated by a self-excited Poisson process, in which case they obey Boltzmann-Gibbs thermodynamics, or by a Critical process, in which long-range interactions in non-equilibrium states are expected (correlation) and the thermodynamics deviate from the Boltzmann-Gibbs formalism. Emphasis is given to background earthquakes since it is generally agreed that aftershock sequences comprise correlated sets. Accordingly, the analysis is based on the accurate earthquake catalogue compiled of the South California Earthquake Data Center, in which aftershocks are either included or have been removed with a stochastic declustering procedure. We examine multivariate cumulative frequency distributions of earthquake magnitudes, interevent time and interevent distance, in the context of Non-Extensive Statistical Physics, which is a generalization of extensive Boltzmann-Gibbs thermodynamics to non-equilibrating (non-extensive) systems. The results indicate a persistent subextensive seismogenetic system exhibiting long-range, moderate to high correlation. Criticality appears to be a plausible causative mechanism although conclusions cannot be drawn until alternative complexity mechanisms can be ruled out.
\end{abstract}

Keywords: Tsallis entropy, complexity, non-extensivity, statistical seismology.

\section{Пврі́र}

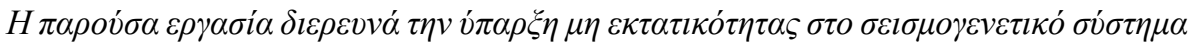

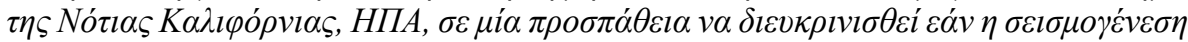

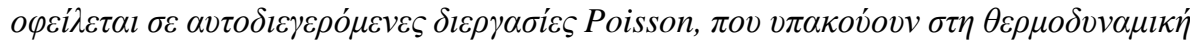

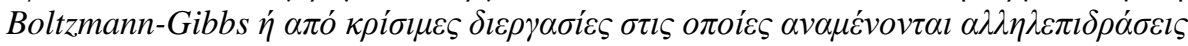

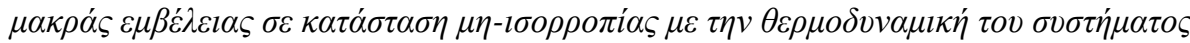



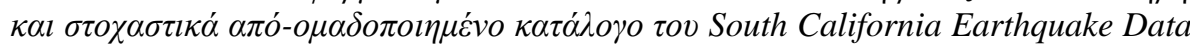

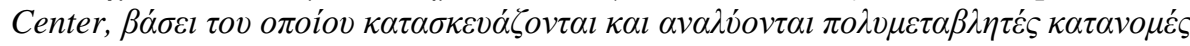

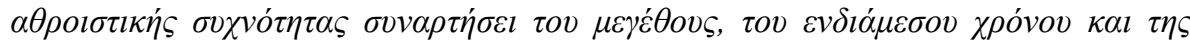

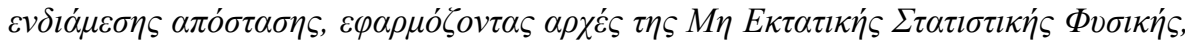

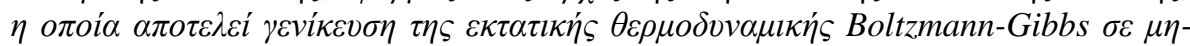

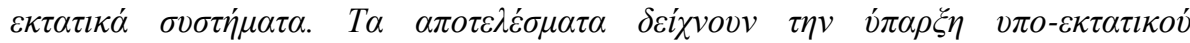

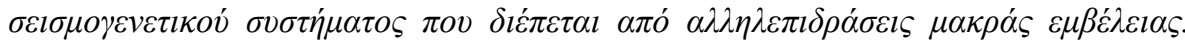

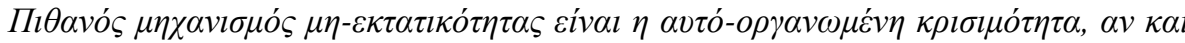




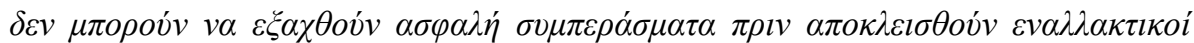

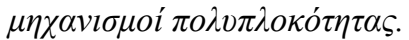



\section{Introduction}

Over the last two decades seismicity modelling with increasing component of physical reasoning has been attracting attention. Statistical seismology comprises a useful tool which aims to bridge the gap between physics-based models without statistics, and statistics-based models without physics. Although progress has been made, seismicity has proved a difficult subject to deal with and make breakthroughs. The seismogenetic system is generally thought to comprise a mixture of processes that express the continuum of tectonic deformation (background process) and a large population of aftershocks that express the short-term activity associated with the occurrence of significant earthquakes (foreground process). Although, progress has been made in understanding the foreground process, the statistical physics of background seismicity, hence the nature of seismogenetic system remains ambiguous with significant repercussions for problems such as hazard analysis and long-term forecasting.

The development of theoretical and experimental methods and techniques that combine the physics and statistics of seismogenesis has led to two principal approaches as to the nature of background seismicity. The first approach postulates that the expression of the background process is Poissonian in time and in space and obeys extensive Boltzmann-Gibbs thermodynamics. This property is associated only with the time and the distance (space) between earthquake events, but not with their size (magnitude) which is governed by the well-established frequency - magnitude (F-M) relationship of Gutenberg and Richter (1944). The second approach proposes that the seismogenetic process comprises a complex system, although the mechanisms begetting complexity are not clear as yet. The first (Poissonian) and currently most influential point of view is supported by a series of well-known models suggesting that background earthquakes are statistically independent and although it is possible for one event to trigger another, this occurs in an unstructured random way and does not to contribute to the long-term evolution of seismicity. Such models include the paradigmatic ETAS (Epidemic Type Aftershock Sequence) which essentially expresses a selfexcited conditional Poisson process, (e.g. Ogata, 1988, 1998; Zhuang et al., 2002; Helmstetter and Sornette, 2003; Touati et al., 2009 and Segou et al., 2013), proxy-ETAS models (Console and Murru, 2001; Console et al., 2003, 2010) the PPE model (Proximity to Past Earthquakes; Marzocchi and Lombardi, 2008), the EEPAS model (Each Earthquake is a Precursor According to Scale; Rhoades, 2007) as well as their variants and derivatives. The second point of view proposes that seismicity is an expression of non-equilibrating fractal tectonic grain that continuously evolves toward a stationary critical state with no characteristic spatiotemporal scale (e.g. Bak and Tang, 1989; Sornette and Sornette, 1989; Olami et al., 1992; Sornette and Sammis, 1995; Rundle et al., 2000; Bak et al., 2002 and Bakar and Tirnakli, 2009, etc.). This concept is known as Self Organized Criticality (SOC) and suggests that all earthquakes evolve towards the same global population and participate in shaping a non-equilibrium state with correlation between background, as well as between background/foreground and foreground/foreground events, so that instabilities arise spontaneously and any small instability has a chance of cascading into a large shock. Correlation endows the seismogenetic system with memory and the statistics of the parameters pertaining to its temporal and spatial evolution are expected to exhibit power-law behaviour and long tails.

Both Poissonian and SOC models agree that the foreground process comprise a set of dependent events, though the former assign only local significance to this dependence, while SOC considers them to be an integral part of the regional seismogenetic process. The fundamental difference between the two approaches lies in their understanding of the background seismogenetic process. It is therefore clear that if it is possible to identify and remove the foreground process (aftershocks), it would also be possible to clarify the nature and dynamics of the background process by examining its spatiotemporal characteristics for the existence of correlation. 
The most recent development in the analysis of earthquake occurrence is the introduction of NonExtensive Statistical Physics (NESP) as a fundamental conceptual framework of the thermodynamics that govern seismogenesis and seismicity. NESP has been developed by Tsallis $(1988,2009)$ as a generalization of the (extensive) Boltzmann-Gibbs formalism which applies to equilibrating physical systems to non-extensive (non-equilibrating) systems. As such it comprises an appropriate tool for the analysis of complexity evolving through scale invariance, long-range interactions and long-term memory (e.g. Gell'mann and Tsallis, 2004). NESP predicts specific power-law cumulative probability distributions for non-extensive (complex) dynamic systems, which reduce to the exponential cumulative distribution in the limiting case of extensive (random) systems. Thus, NESP also provides a unique, consistent and model-independent theoretical context in which to investigate the nature and dynamics of the background or/and foreground seismogenetic processes.

The present study is part of a continued systematic attempt to examine the dynamics of the seismogenetic system by implementing the generalized NESP formalism and searching for signs of randomness or self-organization as a function of event size, time and space. Earthquake magnitude is quite obviously the fundamental measure of event size and the cumulative frequency - magnitude distribution (Gutenberg - Richter law) is understood to express the size distribution of a fractal active fault system. A measure of the temporal correlation between earthquakes can be afforded by the lapse-time between consecutive events over a given area. This parameter is variably referred to as interevent time, waiting time or calm time and understanding its statistics is obviously key to understanding the evolution (temporal dynamics) of the seismogenetic system. Likewise, a measure of spatial correlation (range of interaction) is the hypocentral distance between consecutive events over a given area (interevent distance). It can simply be suggested that if the cumulative frequency distributions of the relevant parameters exhibit specific traits expected of non-extensive systems, then it is likely that the system generating these distributions is non-extensive and vice versa.

The remaining of this presentation is organized as follows. A brief exposé of our implementation of the NESP formalism is given in Section 2. The analysis focuses on South Californian seismicity, because it is a well-studied area with very reliable earthquake monitoring services and seismological catalogues. A brief presentation of the data (earthquake catalogues) and the data reduction and analysis procedures is given in Section 3. The results are presented in Section 4 and discussed in Section 5. Finally, it shall be demonstrated that the frequency of earthquake occurrence in South California is multiply related to the magnitude, interevent time and interevent distance by welldefined bivariate power-laws consistent with NESP and that the expression of the regional active fault system (seismicity) is produced by a complex system comprising a mixture of correlated background and correlated foreground processes, thus displaying attributes of self-organization.

\section{Non- Extensive statistical Physics (NESP) approach to earthquake statistics}

During the past two decades, it has been widely appreciated that in a wide spectrum of complex dynamic natural, physical and social systems, the entropy of the system does not equal the sum of the entropy of their components. Such non-additive systems, also commonly known as nonextensive, are generally characterized by scale invariance, long-range interactions, long-term memory and evolution in a fractal-like space-time: they cannot be adequately described with the standard Boltzmann-Gibbs formalism of thermodynamics, which has been specifically developed for additive (extensive) systems.

The development of an appropriate thermodynamic description of non-extensive systems has been pioneered by Tsallis, $(1988,2009)$ who introduced the context of Non Extensive Statistical Physics (NESP) as a direct generalization of the BG formalism. Thus, letting $X$ be some dynamic parameter, the nonequilibrium states of non-extensive systems can be described by the Tsallis (1988) entropic functional: 
$S_{q}=k \frac{1}{q-1}\left[1-\int_{W} p^{q}(X) d X\right]$,

where $p(X) d X$ is the probability of finding its value in $[X, X+d X]$ so that $\int_{W} p(X) \mathrm{d} X=1, \mathrm{k}$ is the Boltzmann constant and $q$ is the entropic index. The latter is a measure of the non-extensivity of the system and for $q=1$ Eq. 1 reduces to the well-known Boltzmann-Gibbs entropic functional $S_{B G}=-k \int_{W} p(X) \ln (p(X)) d X$

It can be shown (Tsallis, 1988, 2009 and Abe and Suzuki, 2005) that if the empirical distribution of $X$ (escort probability) is $P_{q}(X)$, then the cumulative probability function (CDF) of $X$ is:

$$
P(>X)=\int_{X}^{\infty} d X P_{q}(X)
$$

In the case of $q>0$ and $X \in[0, \infty)$, the CDF reduces to the $q$-exponential distribution

$$
P(>X)=\int_{X}^{\infty} d X P_{q}(X) \ldots \Rightarrow \ldots P(>X)=\exp _{q}\left(-\frac{X}{X_{0}}\right)=\left[1-(1-q)\left(\frac{X}{X_{0}}\right)\right]^{\frac{1}{1-q}}
$$

where $X_{0}$ is a characteristic value ( $q$ - relaxation value) of the seismic parameter and

$$
\exp _{q}(x)=\left\{\begin{array}{ll}
(1+(1-q) x)^{\frac{1}{1-q}} & 1+(1-q) x>0 \\
0 & 1+(1-q) x \leq 0
\end{array},\right.
$$

is the $q$-exponential function which comprises a generalization of the exponential function: for $q=1$, $\exp _{q}(x)=\mathrm{e}^{x}$.

As is apparent in Eq. 2 and Eq. 3, $P(>X)$ is a power-law with a long tail if $q>1$, corresponding to $s u b$ additivity (sub-extensivity), an exponential distribution if $q=1$, corresponding to additivity (extensivity), and a cut-off if $0<q<1$, corresponding to super-additivity (super-extensivity). Accordingly, when $q>1$ the tail of the CDF indicates a complex system with long-range correlations and long-term memory; when $q=1$, the $q$-exponential distribution reduces to the common exponential distribution indicating that the system is a random process. Finally, when $q<1, P(>X)=0$ whenever the argument becomes negative and the system is characterized by a bounded correlation radius.

The analysis of one-dimensional Frequency-Interevent Time (F-T) and Frequency-Magnitude (FM) distributions under the scope of NESP has been attempted by a significant number of researchers. This includes theoretical studies of emergent $q$-exponential distributions in critical (e.g. Caruso et al., 2007 and Bakar and Tirnakli, 2009) and non-critical seismicity models (e.g. Celikoglu et al., 2010), as well as empirical studies in rock fracture experiments (Vallianatos et al., 2012). It also includes the analysis of observed F-T distributions, in which empirical $P(>T)$ functions where effectively fitted with one-dimensional $q$-exponentials (Abe and Suzuki, 2005; Carbone, 2005, Vallianatos et al., 2013; Vallianatos and Sammonds, 2013; Papadakis et al., 2013 and Michas et al. 2013). Analogous studies of F-M distributions have been undertaken by Sotolongo-Costa and Posadas (2004), Silva et al. (2006), Telesca $(2011,2012)$ etc. These authors proposed NESP compatible formulations based on physical models of faults that consider the interaction between two rough fault walls (asperities) and the fragments filling space between them (fragment-asperity model). Energy is stored in the asperities and fragments and their interaction is supposed to modulate earthquake triggering. In this study we posit that the model proposed by Telesca $(2011,2012)$, i.e. that the energy scales with the area of the fragments and asperities $\left(E \propto r^{2}\right)$ so that $M=2 / 3 \log (E)$, is representative of the seismogenetic process. Accordingly, we adopt this model, in which the cumulative F-M distribution reads: 
$P(>M)=\frac{N(>M)}{N_{0}}=\left(1-\frac{1-q_{M}}{2-q_{M}} \cdot \frac{10^{M}}{\alpha^{2 / 3}}\right)^{\left(\frac{2-q_{M}}{1-q_{M}}\right)}$

where $N$ is the number of earthquakes with magnitude greater than $M, N_{0}$ is the total number of earthquakes at $M=0, \alpha$ expresses the proportionality between the released energy $E$ and the fragment size $r$ and $q_{M}$ is the entropic index.

As mentioned in the introduction, our goal is to investigate whether seismicity is generated by a critical, or self-excited random (Poissonian) system. One path to this end is to investigate whether earthquakes are simultaneously correlated in space and in time using the NESP formalism and determining the values and variation of the entropic indices. Accordingly, the earthquake occurrence model we implement herein is based on the multivariate frequency distributions that express the joint probability of observing an earthquake of given magnitude above a given interevent time and/or interevent distance. Specifically, we use the F-M-T distribution constructed as follows: A threshold (cut-off) magnitude $M_{t h}$ is set and a bivariate frequency table $H$, representing the incremental distribution, is first compiled. The cumulative distribution is then obtained from the incremental distribution by backward bivariate summation, according to the scheme

$$
N_{m \tau}=\sum_{j=D_{T}}^{\tau} \sum_{i=D_{M}}^{m}\left\{H_{i j} \Leftrightarrow H_{i j} \neq 0\right\}, \quad \tau=1, \ldots D_{T}, \quad m=1, \ldots D_{M}
$$

where $D_{M}$ is the dimension of $H$ along the magnitude axis and $D_{T}$ is the dimension of $H$ along the interevent time axis. In this construct, the cumulative frequency (earthquake count) can be written thus: $N\left(\left\{M \geq M_{t h}, T: M \geq M_{t h}\right\}\right)$. Then, the empirical probability $P\left(>\left\{M \geq M_{t h}, T: M \geq M_{t h}\right\}\right)$ is simply $\frac{N\left(>\left\{M \geq M_{t h}, T: M \geq M_{t h}\right\}\right)}{N_{0}}, \quad N_{0}=N\left(M=M_{t h}, 0\right)=\|N\|_{\infty}$

Next, we assume that the magnitude $M$, interevent time $\Delta t$ distributions are due to independent processes in the sense that the joint probability $P(M \cup \Delta t)$ factorizes into the probabilities of $\mathrm{M}$ and $\Delta t$, i.e. $P(M \cup \Delta t)=P(M) P(\Delta t)$. Then, on removing the normalization, the joint probability above a magnitude threshold can be expressed by

$$
\log N=\log \left(N_{M=0}\right)+\left(\frac{2-q_{M}}{1-q_{M}}\right) \cdot \log \left(1-\frac{1-q_{M}}{2-q_{M}} \cdot \frac{10^{M}}{\alpha^{2 / 3}}\right)+\frac{1}{1-q_{t}} \log \left[1-\left(1-q_{t}\right)\left(\frac{\Delta t}{\Delta t_{0}}\right)\right],
$$

where $q_{M}, q_{t}$ are the entropic indices for the magnitude and interevent times respectively and $\Delta t_{0}$, is the q-relaxation time, analogous to the relaxation (characteristic) time often encountered in the analysis of physical systems. Eq. 5 is a generalized (bivariate) law of the Gutenberg - Richter kind, in which $b_{q}=\left(2-q_{M}\right)\left(q_{M}-1\right)^{-1}$ is the NESP equivalent of the $b$ value. Accordingly, Eq. 5 is the general model to be implemented in the ensuing analysis.

Eq. 5 is fitted to the observed bivariate F-M-T distributions using non-linear least-squares. Because the parameters of Eq. 5 are subject to positivity constraints and/or are bounded (e.g. the entropic indices), a NNLS solver implementing the trust-region reflective algorithm (e.g. Moré and Sorensen, 1983; Steihaug, 1983) was chosen, together with Least Absolute Residual minimization so as to down-weight possible outliers. Typical examples/results of the performance of this procedure can be found in Tzanis et al. (2013) and Efstathiou et al. (2015) and will not be included herein for the sake of brevity.

As repeatedly stated, complexity and self-organization are associated with long-range interactions and long-term memory; these attributes are referred as correlation. Conversely, Poissonian processes are local and memory-less: they are uncorrelated. The study of F-M-T distributions by means of Eq. 5 will provide information with respect to the size distribution and memory of the seismogenetic system, but not with respect to long-range spatial correlation. In order to address this problem we use the interevent distance, i.e. the hypocentral distance between consecutive earthquakes, as a spatial filter by which to separate and study the temporal correlation of proximal and distal earthquakes: the premise is that if distal earthquakes are correlated in time, then they have to be correlated in space by long-distance interaction and vice versa. To this effect, we apply the same 
modelling procedure to data subsets grouped according to the interevent distance following the rule $C \supset\left\{C_{D}: M>M_{t h} \wedge \Delta d_{L} \leq \Delta d \leq \Delta d_{U}\right\}$ where $C$ is the catalogue, $C_{D}$ is the subset catalogue, $\Delta d$ is the interevent distance and $\Delta d_{L}, \Delta d_{U}$ are the upper and lower group limits.

\section{Earthquake Catalogues and Analysis}

The earthquake data utilized in this study was extracted from the regional earthquake catalogue of South California Earthquake Data Centre (SCEDC @ http://www.data.scec.org). The catalogue comprises 16,749 events recorded between 1981 and 2010 in the area $36^{\circ} \mathrm{N}-32^{\circ} \mathrm{N}$ and $-122^{\circ} \mathrm{E}-$ $114^{\circ} \mathrm{E}$. These geographic boundaries where defined on the basis of the right-lateral active faulting system of South California, which extends between the Salton Sea to the south and the Garlock fault to the north (see Fig. 1a). The area spanned by the WNW-ESE oriented Garlock Fault is not included in the present analysis: it is characterized by a mixture of reverse and left-lateral strike slip faulting, while the Garlock Fault is a major boundary between the semi-rigid microplate of Basin and Range Province and the Mojave Desert and is believed to have developed in order to accommodate the strain differential between the extensional tectonics of the Great Basin crust and the right lateral strike-slip faulting of the Mojave Desert crust. We consider that the expression of seismicity in this area has to be studied separately as it represents a significant and distinct geodynamic feature.

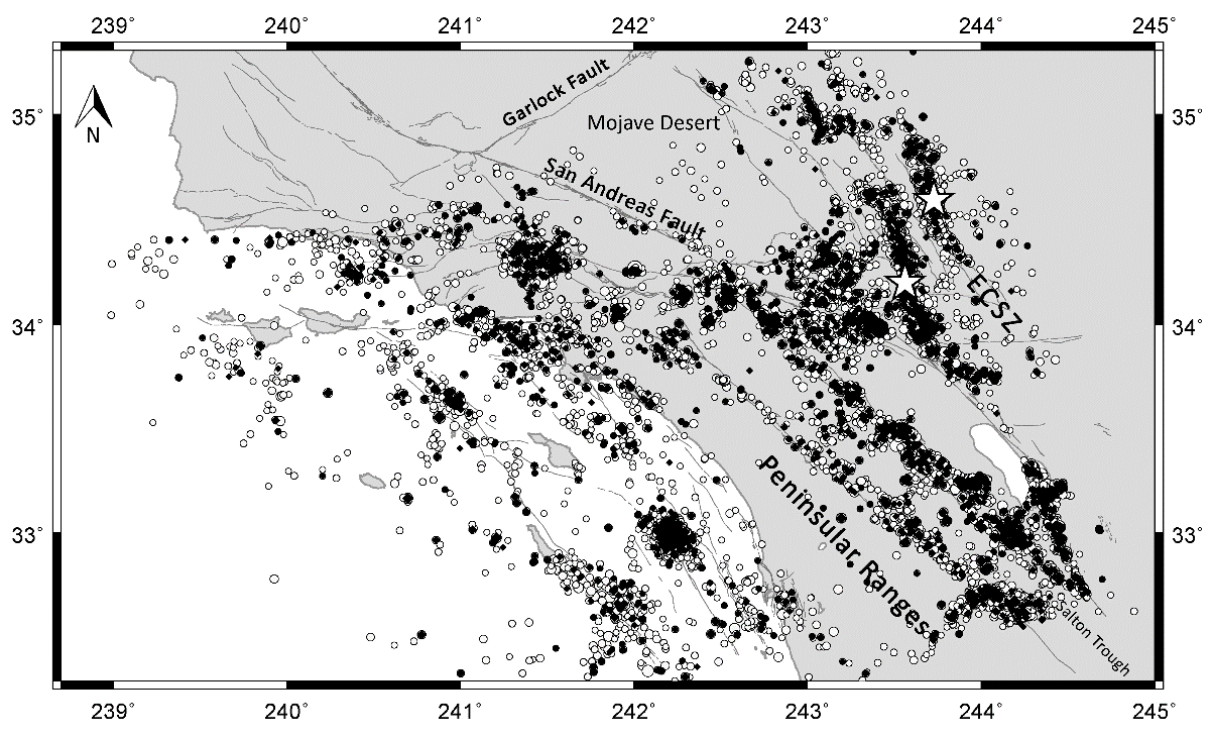

Fig 1a - The SCEDC catalogue, 1981-2010: Epicentral distribution of the complete (white circles) and declustered catalogues (black circles).

Most earthquakes in the SCEDC catalogue are reported in the $M_{L}$ and $M_{w}$ magnitude scales while there is a considerable number of events reported in the duration $\left(M_{d}\right)$ and amplitude $\left(M_{x}\right)$ scales. The latter have been exhaustively calibrated against the $M_{L}$ scale: Eaton (1992) has shown that they are within $5 \%$ of the $M_{L}$ scale for magnitudes in the range 0.5 to 5.5 and that they are virtually independent of the distance from the epicentre to at least $800 \mathrm{~km}$. In consequence, $M_{d}$ and $M_{x}$ are practically equivalent to $M_{L}$. For the purpose of the present analysis $M_{w}$ magnitudes were converted to $M_{L}$ using the empirical formula of Uhrhammer et al. (1996): $M_{\mathrm{w}}=M_{L} \cdot(0.997 \pm 0.020)-(0.050 \pm$ 0.131). The reduced SCEDC catalogue was determined to be complete for magnitudes $M_{L} \geq 2.6$ since 1975; the distribution of the epicentres of all events above the magnitude of completeness is shown in Fig. 1a and the corresponding cumulative earthquake count in Fig. 1b (black line).

As stated in the introduction, it is not clear whether the background seismogenetic process is fundamentally random or correlated. In order to address this question we conduct our analysis on 
both complete (original) and declustered versions of the SCEDC catalogue (in which aftershock sequences have been eliminated in an optimal as possible way). To this effect, we apply the stochastic declustering method of Zhuang et al. (2002) which is based on space-time branching approaches to describe how each event triggers its successors. This method was chosen over deterministic methods (e.g. Gardner and Knopoff, 1974; and Reasenberg, 1985) because the choice of the space-time distance between events is optimized by fitting an ETAS model to the earthquake data and there is no need to assume anything about the parameters pertaining to the definition of the space-time distance. Moreover, instead of associating an aftershock to only one main shock, the method assigns each earthquake with a probability that it is an aftershock of each preceding earthquake, meaning that all preceding earthquakes are possible main shocks of the events that follow them. This is advantageous in that it circumnavigates the difficulty of making committing binary decisions in the (very frequent) case of nearly equal space-time distances between successive events (as with deterministic declustering methods). Last, but not least, the Zhuang et al. method is based on the paradigmatic realization of the self-excited Poisson process (ETAS): if background seismicity obeys Boltzman-Gibbs statistics, then this method should be able to extract a nearly random background process against which to test the alternative hypotheses.

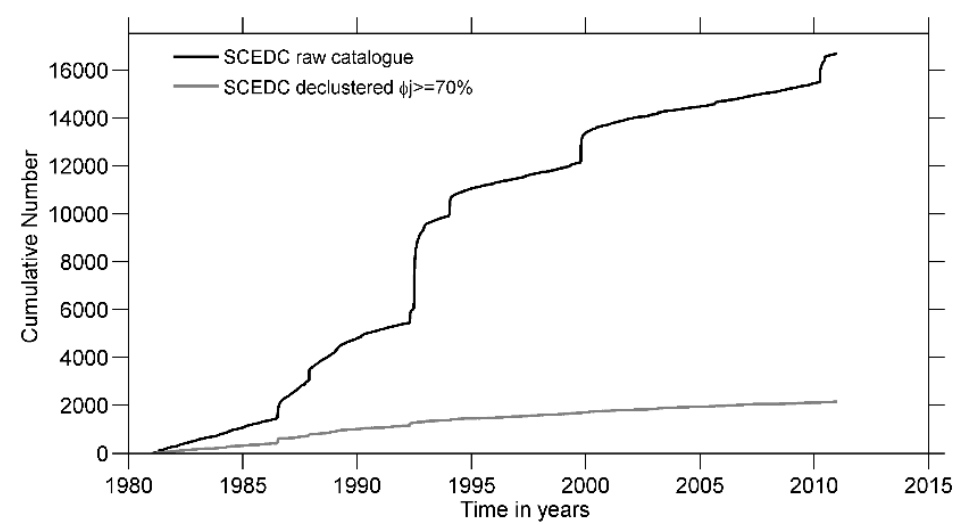

Fig 1 b - Cumulative earthquake count for the complete (solid black line) and declustered catalogues (solid grey line).

The Zhuang et al. (2002) method uses the following form of the normalized probability that one event will occur in the next instant, conditional on the hitherto history of the seismogenetic process (conditional intensity):

$\lambda\left(t, x, y, M \mid H_{t}\right)=\mu(x, y, M)+\sum_{i: t_{i}<t} \kappa\left(M_{i}\right) \cdot g\left(t-t_{i}\right) \cdot f\left(x-x_{i}, y-y_{i} \mid M_{i}\right) \cdot j\left(M \mid M_{i}\right)$

where, $\lambda$ is the conditional intensity on the history of observation $H_{t}$ until time $t, \mu(x, y, M)$ is the background intensity, $\kappa(M)$ is the expected number of foreground events triggered by a magnitude $M$ main shock and $g(t), f\left(x, y \mid M_{i}\right)$ and $j\left(M \mid M_{i}\right)$ are respectively the probability distributions of the occurrence time, the location and the magnitude events triggered by a main shock of magnitude $M_{i}$. If the catalogue is arranged in chronological order, then the probability of an event $j$ to have been triggered by an event $i<j$ can be estimated from the occurrence rate at its occurrence time and location as

$$
p_{i, j} \frac{\kappa\left(M_{i}\right) \cdot g\left(t_{j}-t_{i}\right) \cdot f\left(x_{j}-x_{i}, y_{j}-y_{i} \mid M_{i}\right)}{\lambda\left(t_{j}, x_{j}, y_{j} \mid H_{t}\right)}
$$

and the probability that an event $j$ is aftershock is given by $p_{j}=\sum_{i=1}^{j-1} p_{i, j}$. Conversely, the probability that an event $j$ is background is given by $\phi_{j}=1-p_{j}$. 
As a rule of thumb, an event with $\phi_{j} \leq 50 \%$ is taken to be foreground while an event with $\phi_{j}>50 \%$ is likely to belong to the background. In this presentation, we assume that events with probability $\phi_{j}$ $>70 \%$ belong to the background. Accordingly, the declustered version of the catalogue we use herein comprises 2,158 events with probability $\phi_{j}>70 \%$ to belong to the background. The epicentre distribution of the declustered catalogue is also shown in Fig 1a and the corresponding cumulative earthquake count in Fig 1b (grey line).

\section{Results}

As a general rule, Eq. 5 yields excellent approximations of the observed F-M-T distributions (for details see Tzanis et al., 2013 and Efstathiou et al., 2015). For the sake of experimental rigour, this presentation will only consider models associated with a goodness of fit $\left(R^{2}\right)$ better than 0.97 . The discussion will focus on the values and variation of the entropic indices determined for different cutoff magnitudes (Fig. 2) and interevent distances (Fig 3). The following two conclusions of Efstathiou et al. (2015) are important in studying and comprehending the results obtained herein:

(a) The estimation of the $b$-value from the magnitude entropic index $\left(b_{q}\right)$ is robust and absolutely consistent with the $b$-value obtained by standard methods of b-value estimation.

(b) The analysis of a large number of synthetic ETAS catalogues based on the parameterization of Californian seismicity has established that the threshold (base) value of the temporal entropic index above which it is safe to assume non-Poissonian background processes, is $q_{T}=1.2$.
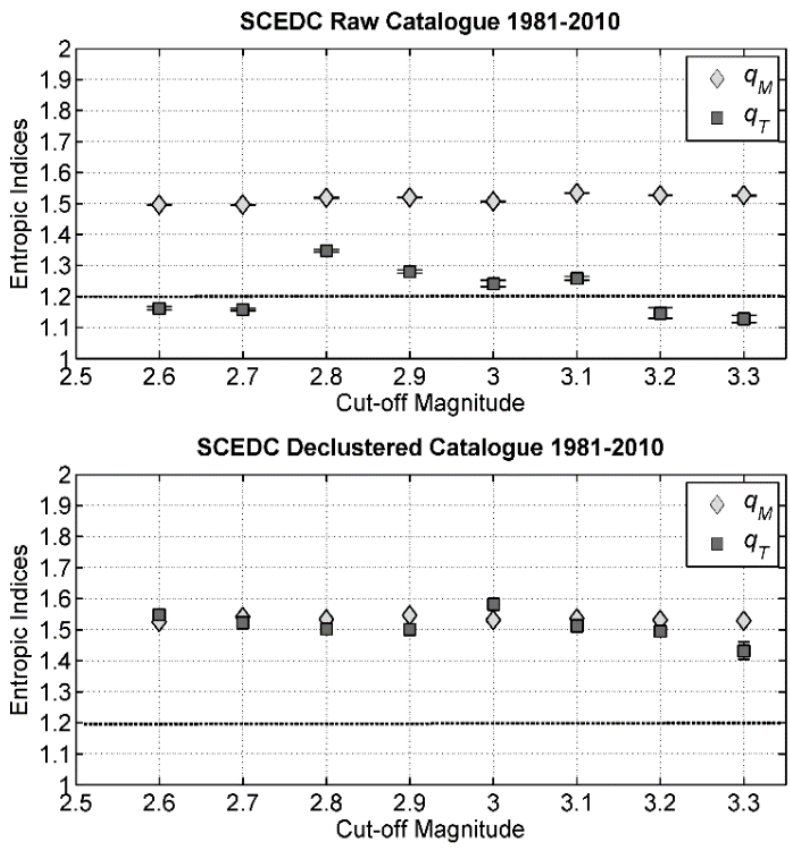

Figure 2 - Dependence of entropic indices on the threshold magnitude for the complete (raw) SCEDC catalogue (top) and the declustered catalogue at the $70 \%$ probability level (bottom). In all cases, error bars correspond to $95 \%$ confidence intervals.

As is apparent in Fig. 2, the analysis of the complete and declustered SCEDC catalogue shows that the entropic index $q_{M}$ is quite consistently determined. For the complete catalogue $q_{M}$ varies from 1.5 to 1.53 while for its declustered counterpart it varies from 1.52 to 1.54 . This leads to $b_{q}$ estimates that vary from 1.00 to 0.88 for the complete catalogue, and 0.92 to 0.85 for its declustered counterpart. The entropic index $q_{M}$, like the $b$-value to which it is related, represents the scaling of the size distribution of earthquakes and clearly indicates a correlated, scale-free process. The 
temporal entropic index exhibits significant variations for the complete SCEDC catalogue. As can be seen in the top panel of Fig. 2, $q_{T}$ varies from 1.15 to 1.34 indicating very low to moderate correlation; at first sight, it would appear that the seismogenetic system of South California verges on the border between point and critical processes. Conversely, the declustered SCEDC catalogue reserves a surprise (Fig. 2-bottom): $q_{T}$ is consistently stable and varies from 1.43 to 1.58 indicating a highly correlated background process at the $70 \%$ probability level.

When interevent distances are used as spatial filters, the analysis shows that $q_{M}$ is also stable and varies from 1.49 to 1.52 for the complete SCEDC catalogue, and from 1.51 to 1.53 for the declustered version. Respectively, $b_{q}$ varies from 1.04 to 0.92 and from 0.96 to 0.88 . Conversely, the temporal entropic index changes with interevent distance. Fig. 3-top clearly shows that for the complete catalogue, $q_{T}$ is very high (1.78) at shorter interevent distances $(\Delta d \leq 50 \mathrm{~km})$ indicating extremely high correlation which is evidently due to the overwhelming effect of the tightly spaced and highly correlated aftershock sequences. For $\Delta d>100 \mathrm{~km} q_{T}$ is persistently lower (1.31-1.29) indicating moderately correlated farfield processes. The analysis of the declustered SCEDC catalogue shows that $q_{T}$ behaves in a manner analogous to the analysis of its complete counterpart: as evident in Fig. 3-bottom, $q_{T}$ indicates extremely high correlation (1.91) at short interevent distances $(\Delta d \leq 50 \mathrm{~km})$, while it varies from 1.35 to 1.48 at longer interevent distances indicating moderate to high far-field correlation.
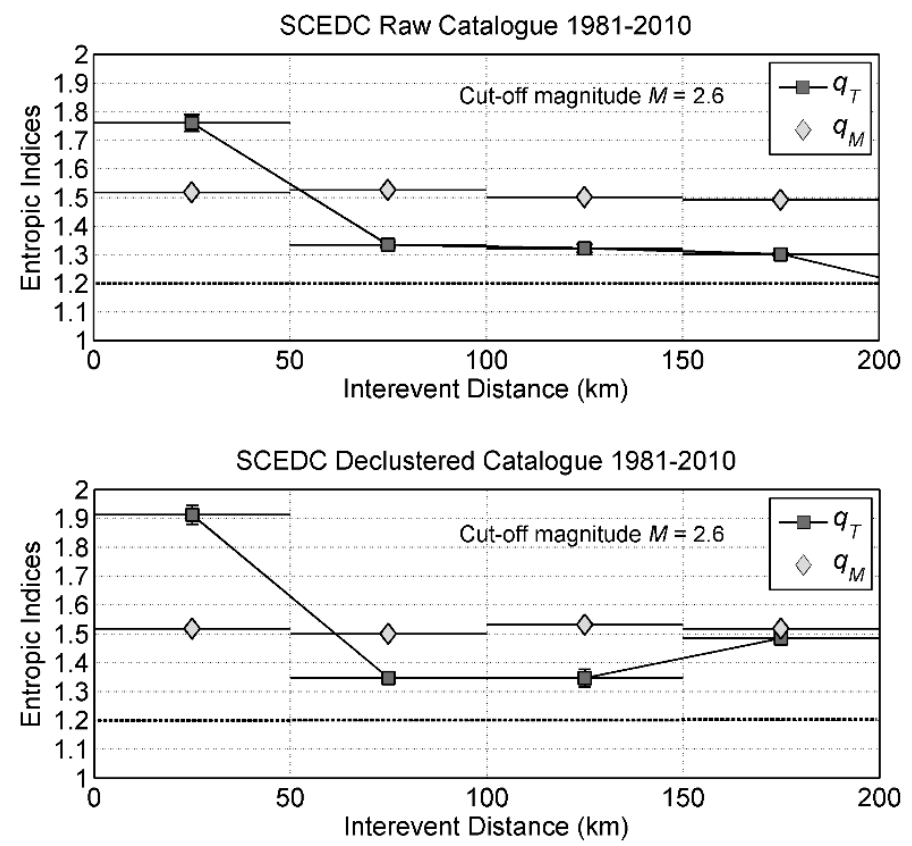

Figure 3 - The dependence of the entropic indices on interevent distance, for data subsets grouped according to interevent distance. (a) Analysis of the complete (raw) catalogue. (b) Analysis of the declustered catalogue at the $70 \%$ probability level. The size of $\Delta d$ bins is indicated by horizontal line segments. Errors are $95 \%$ confidence intervals.

\section{Discussion}

The present study is part of a continued systematic attempt to examine the nature of seismogenesis by searching for evidence of complexity and non-extensivity in accurate and reliable seismicity records. There are two general theoretical approaches as to the nature of the background seismogenetic process: either it comprises a self-excited conditional Poisson process, or a (SelfOrganized) Critical process. In the first case, background (core) earthquakes are thought to be spontaneously generated in the seismogenetic continuum and are expected to be independent of each 
other. Accordingly, there should be no correlation between background events as there is no interaction between them and the thermodynamics of the process should obey the Boltzmann-Gibbs entropic formalism. In the latter case, long-range interactions in non-equilibrium states are expected, so that background events should be correlated and the thermodynamics should deviate significantly from the Boltzmann-Gibbs formalism. Both approaches agree that the core earthquakes generate aftershock sequences (foreground earthquakes) which are genetically related to the parent event and comprise correlated sets. It is clear that if it was possible to identify and remove aftershocks, it would also be possible to investigate the nature of the seismogenetic system by examining background earthquakes for the existence of correlation.

With respect to South Californian seismicity the analysis of size (energy) distribution of earthquakes yielded results consistent with a correlated sub-extensive system; the results are also compatible with observations based on conventional measures of scaling and self-organization, with particular reference to the $b$-value. More interesting observations can be made in reference to the temporal entropic index $q_{T}$, which conveys information about the dynamic state(s) of the system. For the complete catalogue, the analysis of interevent times has determined an overall moderately correlated sub-extensive system, in which near-field events (at short interevent distance) exhibit very high correlation due to the interactions between proximal background/background, background/foreground and foreground/foreground events. A point of great significance is that on removing aftershock sequences we observe increased correlation. The background process, with probability $\phi_{j} \geq 70 \%$ for an event to belong to the core seismicity appears to be highly correlated in both time and space (i.e. at both near and far fields) while properly random processes $\left(q_{T}<1.1\right)$ have not been detected.

The results presented herein provide strong evidence of complexity in the expression of background seismicity in South California. Criticality appears to be a very likely explanation of the complexity mechanism, inasmuch as a persistent state of non-equilibrium can be inferred on the basis of the temporal entropic index and persistent fractal geometry is evident in the behaviour of the magnitude entropic index. However, supplementary experimental work and testing is required before the evidence becomes compelling. According to Sornette and Werner (2009), complexity may not only emerge from inherent non-linear dynamics of the active tectonic grain as required by SOC; quenched heterogeneity in the stress field and production rates may also be of great importance. It is also noteworthy that Celikoglu et al. (2010) showed that it is possible to obtain $q$-exponential distributions of interevent times with models not involving criticality. Accordingly, additional work is required before the mechanism of background seismicity can be specified.

As a general conclusion, the NESP formalism, although far from having settled the questions and debates on the statistical physics of earthquakes indicates an excellent natural descriptor of earthquake statistics and appears to apply to the seismicity observed along in South California where sub-extensive thermodynamics appear to predominate.

\section{Acknowledgments}

This work was partly supported by the THALES Program of the Ministry of Education of Greece and the European Union in the framework of the project "Integrated understanding of Seismicity, using innovative methodologies of Fracture Mechanics along with Earthquake and Non-Extensive Statistical Physics - Application to the geodynamic system of the Hellenic Arc - SEISMO FEAR HELLARC".

\section{References}

Abe, S. and Suzuki, N., 2005. Scale-free statistics of time interval between successive earthquakes, Physica A, 350, 588-596.

Bak, P. and Tang, C., 1989. Earthquakes as a self-organized critical phenomenon, J. Geophys. Res., 94, 15635-15637. 
Bak, P., Christensen, K., Danon, L. and Scanlon, T., 2002. Unified scaling law for earthquakes, Phys. Rev. Lett., 88, 178501, doi: 10.1103/PhysRevLett.88.178501.

Bakar, B. and Tirnakli, U., 2009. Analysis of self-organized criticality in Ehrenfest's dog-flea model, Phys. Rev. E., 79, 040103, doi: 10.1103/PhysRevE.79.040103, 2009.

Carbone, V., Sorriso-Valvo, L., Harabaglia, P. and Guerra, I., 2005. Unified scaling law for waiting times between seismic events, Europhys. Lett., 71(6), 1036, doi: 10.1209/epl/i2005-10185-0.

Caruso, F., Pluchino, A., Latora, V., Vinciguerra, S. and Rapisarda, A., 2007: Analysis of self-organized criticality in the Olami-Feder-Christensen model and in real earthquakes, Phys. Rev. E, 75, 055101.

Celikoglu, A., Tirnakli, U. and Duarte Queirós, S., 2010: Analysis of return distributions in the coherent noise model, Phys. Rev. E., 82, 021124, doi: 10.1103/PhysRevE.82.021124.

Console, R. and Murru, M., 2001. A simple and testable model for earthquake clustering, J. Geoph. Res., 106, B5, 8699-8711.

Console, R., Murru, M. and Lombardi, A.M., 2003. Refining earthquake clustering models, $J$. Geoph. Res., 108, B10, 2468-2476.

Console, R., Jackson, D.D. and Kagan, Y.Y., 2010. Using the ETAS model for catalog declustering and seismic background assessment, Pure Appl. Geoph., 10.1007/s00024-010-0065-5.

Eaton, J.P., 1992. Determination of amplitude and duration magnitudes and site residuals from shortperiod seismographs in Northern California, Bull. Seism. Soc. Am., 82(2), 533-579.

Efstathiou A., Tzanis A. and Vallianatos, F., 2015. Evidence of Non-Extensivity in the evolution of seismicity along the San Andreas Fault, California, USA: An approach based on Tsallis Statistical Physics, Physics and Chemistry of the Earth, Parts A/B/C, in press, doi: 10.1016/j.pce.2015.02.013.

Gardner, J.K. and Knopoff, L., 1974. Is the sequence of earthquakes in Southern California, with aftershocks removed, Poissonian? Bull. Seism. Soc. Am., 64(5), 1363-1367.

Gell-Mann, M. and Tsallis, C., eds., 2004. Nonextensive Entropy - Interdisciplinary Applications, Oxford University Press, New York.

Gutenberg, B. and Richter, C.F., 1944. Frequency of earthquakes in California, Bull. Seismol. Soc. Am., 34-4, 185-188.

Helmstetter, A. and Sornette, D., 2003. Predictability in the Epidemic-Type Aftershock Sequence model of interacting triggered seismicity, J. Geophys. Res., 108(B10), 2482.

Marzocchi, W. and Lombardi, A.M., 2008. A double branching model for earthquake occurrence, J. Geophys. Res., 113, B0 8317, doi: 10.1029/2007JB005472.

Michas, G., Vallianatos, F. and Sammonds, P., 2013. Non-extensivity and long-range correlations in the earthquake activity at the West Corinth rift (Greece), Nonlinear Proc. Geoph., 20, 713-724.

Moré, J.J. and Sorensen, D.C., 1983. Computing a Trust Region Step, SIAM Journal on Scientific and Statistical Computing, 3, 553-572.

Ogata, Y., 1988. Statistical models for earthquake occurrences and residual analysis for point processes, Journal of American Statistical Association, Application, 83(401), 9-27.

Ogata, Y., 1998. Space-time point-process models for earthquake occurrences, Annals of the Institute of Statistical Mathematics, 50(2), 379-402.

Olami, Z., Feder, H.J.S. and Christensen, K., 1992. Self-Organized Criticality in a continuous, nonconservative cellular automaton modeling earthquakes, Phys. Rev. Lett., 68, 1244-1247.

Papadakis, G., Vallianatos, F. and Sammonds, P., 2013. Evidence of Nonextensive Statistical Physics behaviour of the Hellenic Subduction Zone seismicity, Tectonophysics, 608, 1037-1048.

Reasenberg, P., 1985. Second-order moment of central California seismicity, 1969-82, J. Geophys. Res., 90, 5479, 5495.

Rhoades, D.A., 2007. Application of the EEPAS model to forecasting earthquakes of moderate magnitude in Southern California, Seismol. Res. Lett., 78(1), 110-115.

Rundle, J.B., Klein, W., Turcotte, D.L. and Malaud, B.D., 2000. Precursory seismic activation and critical point phenomena, Pure appl. Geophys., 157, 2165-2182.

Segou, M., Parsons, T. and Ellsworth, W., 2013. Comparative evaluation of physics-based and statistical forecasts in Northern California, J. Geophys. Res. Solid Earth, 118.

Silva, R., Franca, G.S., Vilar, C.S. and Alcaniz, J.S., 2006. Nonextensive models for earthquakes. Physical Review E, 73, 026102, doi: 10.1103/PhysRevE.73.026102. 
Sornette, A. and Sornette, D., 1989. Self-organized criticality and earthquakes, Europhys. Lett., 9, 197-202.

Sornette, D. and Sammis, C.G., 1995. Complex critical exponents from renormalization group theory of earthquakes: Implications for earthquake predictions, J. Phys., 1, 5, 607-619.

Sornette, D. and Werner, M.J., 2009. Statistical Physics Approaches to Seismicity, in Complexity in Earthquakes, Tsunamis, and Volcanoes, and Forecast, In: Lee, W.H.K., ed., in the Encyclopedia of Complexity and Systems Science, R. Meyers (Editor-in-chief), 7872-7891, Springer, ISBN: 978-0-387-755888-6. arXiv:0803.3756v2 [physics.geo-ph] (last access 20 October 2014).

Sotolongo-Costa, O. and Posadas, A., 2004. Tsalli's entropy: A non-extensive frequency-magnitude distribution of earthquakes. Phys. Rev. Letters, 92(4), 048501, doi: 10.1103/PhysRevLett.92.048501.

Steihaug, T. 1983. The Conjugate Gradient Method and Trust Regions in Large Scale Optimization, SIAM Journal on Numerical Analysis, 20, 626-637.

Telesca, L., 2011. Tsallis-based nonextensive analysis of the Southern California seismicity, Entropy, 13, 1267-1280.

Telesca, L., 2012. Maximum Likelihood Estimation of the Nonextensive Parameters of the Earthquake Cumulative Magnitude Distribution, Bull. Seismol. Soc. Am., 102, 886-891.

Tzanis, A., Vallianatos, F. and Efstathiou, A., 2013. Multidimensional earthquake frequency distributions consistent with non-extensive statistical physics: The interdependence of Magnitude, Interevent Time and Interevent Distance in North California, Bull. Geol. Soc. Greece, 47(3), 1326-1337. http://www.geosociety.gr/ images/news_files/ EGE_XLVII/ Vol_3/ 1326_ Tzanis .pdf (last access 24 October 2015).

Touati, S., Naylor, M. and Main, I.G., 2009. Origin and Nonuniversality of the Earthquake Interevent Time Distribution, Phys. Rev. Letters, 102, 168501, doi: 10.1103/PhysRevLett.102.168501.

Tsallis, C., 1988. Possible generalization of Boltzmann-Gibbs statistics, Journal of Statistical Physics, 52, 479-487, doi: 10.1007/BF01016429.

Tsallis, C., 2009. Introduction to Nonextensive Statistical Mechanics: Approaching a Complex World. Springer Verlag, Berlin, 378 pp.

Uhrhammer, B.R.A., Loper, S.J. and Romanowicz, B., 1996. Determination of local magnitude using BDSN Broadband Records, Bull. Seism. Soc. Am., 86(5), 1314-1330.

Vallianatos, F. and Sammonds, P., 2013. Evidence of non-extensive statistical physics of the lithospheric instability approaching the 2004 Sumatran-Andaman and 2011 Honshu megaearthquakes, Tectonophysics, doi: 10.1016/j.tecto.2013.01.009.

Vallianatos, F. and Telesca, L., eds., 2012. Statistical Mechanics in Earth Physics and Natural Hazards, Acta Geophysica, 60, 499-501.

Vallianatos, F., Benson, P., Meredith, P. and Sammonds, P., 2012. Experimental evidence of nonextensive statistical physics behaviour of fracture in triaxially deformed Etna basalt using acoustic emissions, Eur. Phys. Let. (EPL), 97, 58002.

Vallianatos, F., Michas, G., Papadakis, G. and Tzanis, A., 2013. Evidence of non-extensivity in the seismicity observed during the 2011-2012 unrest at the Santorini volcanic complex, Greece. Nat. Hazards Earth Syst. Sci., 13, 177-185, doi: 10.5194/nhess-13-177-2013.

Zhuang J., Ogata Y. and Vere-Jones, D., 2002. Stochastic declustering of space-time earthquake occurrences, Journal of the American Statistical Association, 97, 369-380. 